\title{
Determination of the Absolute Molar Mass of [Fe-S]-Containing Proteins Using Size Exclusion Chromatography-Multi-Angle Light Scattering (SEC-MALS)
}

\author{
Christophe Velours ${ }^{1,2, *}$, Jingjing Zhou ${ }^{3,+}$, Paolo Zecchin ${ }^{3,+}$, Nisha He ${ }^{3}$, Myriam Salameh ${ }^{4}$, \\ Marie-Pierre Golinelli-Cohen ${ }^{4}$ (D) and Béatrice Golinelli-Pimpaneau ${ }^{3, * \text { (D) }}$
}

check for updates

Citation: Velours, C.; Zhou, J.; Zecchin, P.; He, N.; Salameh, M.; Golinelli-Cohen, M.-P.;

Golinelli-Pimpaneau, B.

Determination of the Absolute Molar Mass of [Fe-S]-Containing Proteins Using Size Exclusion

Chromatography-Multi-Angle Light Scattering (SEC-MALS). Biomolecules 2022, 12, 270. https://doi.org/ $10.3390 /$ biom 12020270

Academic Editor: Elżbieta

Skrzydlewska

Received: 21 December 2021

Accepted: 3 February 2022

Published: 8 February 2022

Publisher's Note: MDPI stays neutral with regard to jurisdictional claims in published maps and institutional affiliations.

Copyright: (C) 2022 by the authors. Licensee MDPI, Basel, Switzerland. This article is an open access article distributed under the terms and conditions of the Creative Commons Attribution (CC BY) license (https:// creativecommons.org/licenses/by/ $4.0 /)$.
1 Fundamental Microbiology and Pathogenicity Laboratory, UMR 5234 CNRS-University of Bordeaux, SFR TransBioMed, 33076 Bordeaux, France

2 Institute for Integrative Biology of the Cell (I2BC), CNRS, Université Paris-Saclay, 91198 Gif-sur-Yvette, France

3 Laboratoire de Chimie des Processus Biologiques, UMR 8229 CNRS, Collège de France, Sorbonne Université, 11 Place Marcelin Berthelot, 75231 Paris, France; jingjing.zhou@college-de-france.fr (J.Z.); paolo.zecchin@college-de-france.fr (P.Z.); nshe1992@163.com (N.H.)

4 Institut de Chimie des Substances Naturelles, Université Paris-Saclay, CNRS, UPR2301, 91198 Gif-sur-Yvette, France; myriam.salameh@agroparistech.fr (M.S.); marie-pierre.golinelli@cnrs.fr (M.-P.G.-C.)

* Correspondence: christophe.velours@u-bordeaux.fr (C.V.); beatrice.golinelli@college-de-france.fr (B.G.-P.)

$\dagger$ These authors contributed equally to this work.

\begin{abstract}
Size Exclusion Chromatography coupled with Multi-Angle Light Scattering (SEC-MALS) is a technique that determines the absolute molar mass (molecular weight) of macromolecules in solution, such as proteins or polymers, by detecting their light scattering intensity. Because SECMALS does not rely on the assumption of the globular state of the analyte and the calibration of standards, the molar mass can be obtained for proteins of any shape, as well as for intrinsically disordered proteins and aggregates. Yet, corrections need to be made for samples that absorb light at the wavelength of the MALS laser, such as iron-sulfur [Fe-S] cluster-containing proteins. We analyze several examples of [2Fe-2S] and [4Fe-4S] cluster-containing proteins, for which various corrections were applied to determine the absolute molar mass of both the apo- and holo-forms. Importantly, the determination of the absolute molar mass of the [2Fe-2S]-containing holo-NEET proteins allowed us to ascertain a change in the oligomerization state upon cluster binding and, thus, to highlight one essential function of the cluster.
\end{abstract}

Keywords: SEC-MALS; size exclusion chromatography; multi-angle light scattering; molar mass; molecular weight; Fe-S cluster; iron-sulfur cluster; [Fe-S]-binding protein; NEET protein

\section{Introduction}

Determining the molar mass (or molecular weight) is a critical step in the biophysical characterization of a newly produced protein. Analytical size-exclusion chromatography (SEC) is commonly used for the determination of the molar mass of proteins and proteinprotein complexes in solution, based on the elution volume and a calibration curve based on protein standards [1]. However, when the protein is not globular or undergoes non-ideal column interactions, such determined molar mass is incorrect. In contrast, Multi-Angle Light Scattering (MALS) is an absolute technique that uses a collimated beam from a laser source to determine the exact mass in solution of proteins, lipids, detergents, nucleic acids, sugars or heterologous complexes and to evaluate their gyration radius [2-5]. The combination of SEC for separating proteins by their hydrodynamic radius, with MALS for determining the molar mass of the eluted protein, constitutes a versatile, reliable means for characterizing solutions of one or more protein species. Moreover, SEC-MALS, in which the measurement is performed at each elution volume, can determine if an eluting 
peak is homogeneous or heterogeneous and distinguish between a fixed molecular weight distribution versus a dynamic equilibrium [6,7]. Finally, this method enables the calculation of the stoichiometry of complexes such as protein-protein $[6,8,9]$ or protein-carbohydrate complexes [10]. However, corrections of the light scattering measurements are required to determine the molar mass of polymers that are fluorescent or that absorb light at the operating laser wavelength. Whereas such corrections were previously described for lignin polymers [11], this topic is not frequently discussed in the literature. Thus, we address the case of iron-sulfur [Fe-S] cluster-containing proteins, which absorb light at the laser wavelength.

[Fe-S] clusters are among the oldest cofactors present in both aerobic and anaerobic organisms [12]. They are formed by ferrous and ferric ions bridged by sulfide ions. The most current forms are the [2Fe-2S] and [4Fe-4S] clusters [13], but more complex assemblies exist $[14,15]$. [Fe-S] clusters, typically coordinated by four cysteines, are known for their capacity to accept and give electrons, as exemplified by complex I of NADH quinone oxidoreductase [16]. The intrinsic chemistry of [Fe-S] clusters allows them to serve as sensors of gaseous (oxygen or NO) or nongaseous molecules (reactive oxygen species or ROS), or as a sensor of the Fe and [Fe-S] cluster content in the cell $[17,18]$. Numerous [Fe-S] proteins are also involved in regulating genetic expression $[19,20]$. For example, fumarate and nitrate reductase (FNR) is an interesting case of a transcription regulator, for which the oligomerization state of the protein changes with the chemical nature of the cluster [21,22]. Indeed, in the absence of $\mathrm{O}_{2}, \mathrm{FNR}$ contains a [4Fe-4S] cluster, which allows the dimerization of the protein, specific binding of the protein to DNA and transcription. Yet, in the presence of $\mathrm{O}_{2}$, the [4Fe-4S] cluster is rapidly converted into a [2Fe-2S] form, leading to the dissociation of the dimer and inactivation of FNR. Finally, some [Fe-S] proteins are enzymes, in which the [4Fe-4S] cluster serves as a cofactor to catalyze a chemical reaction [23], such as aconitase that catalyzes the dehydration of citrate [24,25].

Here we investigate [2Fe-2S]-containing regulatory proteins and [4Fe-4S]-dependent sulfuration and desulfidase enzymes. On the one hand, human CISD2 and mitoNEET are two mammalian NEET proteins anchored in the endoplasmic reticulum and mitochondria membranes, respectively, that possess [Fe-S]-containing cytosolic domains that are very close in sequence and structure [26]. This recently discovered class of [2Fe-2S]-proteins are characterized by atypical cluster coordination involving one histidine and three cysteines. Several studies have demonstrated their involvement in the regulation of iron and ROS homeostasis in cells [27]. One of their remarkable properties is their ability to transfer their cluster to a recipient protein under the strict control of the redox state of their cluster [28] and to be able to sense their environment [29]. On the other hand, sulfuration enzymes and desulfidases are [4Fe-4S]-dependent enzymes. tRNA sulfuration enzymes are involved in the biosynthesis of sulfur-containing nucleosides, which are essential for the efficiency and accuracy of genetic translation [30-33], whereas LarE catalyzes sulfur insertion in the cofactor of lactate racemase [34], TudS is a recently discovered thiouracil desulfidase [35] and CyuA is a cysteine desulfidase [36]. In the case of these sulfurases and desulfidases, the [4Fe-4S] cluster is bound by three ligands only, and the fourth non-protein bonded unique iron is presumed to bind and activate the sulfur donor for the sulfuration reaction [30,31] or the sulfur of the substrate for the desulfuration reaction [35].

The determination of the absolute molar mass is an important step, not only for the characterization of these [Fe-S]-binding proteins but also to reveal the potential involvement of the iron-sulfur cluster in protein oligomerization. In SEC-MALS, the SEC column is used solely to separate the various species according to their hydrodynamic radius so that they enter the MALS and concentration detector devices individually, the actual retention time not being significant for the analysis. The calibration of the instruments is independent on the column and does not rely on reference standards. Hence, SEC-MALS is considered an 'absolute' method to determine the molar mass from basic equations, using the ratio of the scattered and incident light intensities at several angles and the sample concentration determined by the refractive index (RI) detector. We detail here a correction method for 
analysis of SEC-MALS data of several [2Fe-2S]- and [4Fe-4S]-containing proteins, collected with the mini-DAWN TREOS Wyatt devices, that can be applied to any [Fe-S]-containing protein and, more widely, to any protein that absorbs at the laser wavelength. We show that the molar mass of the holo-proteins can be determined more accurately than without the correction and that, in the case of two NEET proteins, the binding of the cluster triggers the dimerization of the proteins.

\section{Materials and Methods}

Purification of human CISD2, Escherichia coli MnmA, Aeromonas TudS and human mitoNEET has been described previously [26,32,35,37], whereas the overexpression and purification of LarE and CyuA from Methanococcus maripaludis will be reported elsewhere (unpublished results). MitoNEET and CISD2 were purified aerobically as holo-proteins, whereas chemical cluster reconstitution was performed anaerobically with the as-purified proteins (mixture of apo/holo forms) of MnmA, TudS, LarE and CyuA, as described previously [32]. After anaerobic purification, these latter holo-proteins with labile [Fe-S] clusters were frozen and kept in air-free sealed tubes.

The as-purified MnmA, CyuA and LarE proteins contained such a low amount of cluster $(<5 \%)$ that they were used as the apo-proteins. To obtain the apo-proteins of the NEET proteins, the cluster was removed by incubation of the holo-proteins in the presence of $10 \mathrm{mM}$ ethylenediaminetetraacetic acid (EDTA) and $10 \mathrm{mM}$ dithiothreitol (DTT) until the complete disappearance of the protein color and absorbance in the 320-600 nm range. Attempts to obtain the apo-form of TudS led to the aggregation of the protein.

As a final step of purification, all proteins were loaded on a gel filtration column (Hiload 26/60 Superdex 75 (Cytiva, Marlborough, MA, USA) for NEET proteins, Hiload 26/60 Superdex 200 (Cytiva) for all other proteins equilibrated with the same buffer as used for SEC-MALS analysis; the major eluted peak was collected. Including DTT at a high concentration in the final chromatographic step of the apo-proteins is essential to avoid the formation of intermolecular disulfide bonds between solvent-exposed cysteines.

Proteins were then analyzed with two different types of SEC-MALS/UV/RI light scattering equipment with very similar components: high pressure liquid chromatography (HPLC) from Agilent (Santa Clara, CA, USA) or Shimadzu (Tokyo, Japan), mini-DAWN TREOS and refractometer (Optilab T-rEX or Optilab), all from Wyatt Technology (Santa Barbara, CA, USA). For each sample, $100 \mu \mathrm{L}$ at $\sim 2 \mathrm{mg} \cdot \mathrm{mL}^{-1}$ was injected on the HPLC system equipped with a Superdex 200 increase 10/300 GL column (Cytiva), at a flow rate of $0.5 \mathrm{~mL} \cdot \mathrm{min}^{-1}$ in $50 \mathrm{mM}$ sodium phosphate $\mathrm{pH} 7.5,50 \mathrm{mM} \mathrm{NaCl}, 10 \mathrm{mM}$ DTT (for the [2Fe-2S] proteins) or $25 \mathrm{mM}$ HEPES pH 7.5, $300 \mathrm{mM} \mathrm{NaCl}, 5 \mathrm{mM}$ DTT (for the [4Fe-4S] proteins). The protein concentration was determined using an average refractive index increment $(\mathrm{dn} / \mathrm{dc})$ of $0.183 \mathrm{~mL} \cdot \mathrm{g}^{-1}$ at the laser wavelength $(658 \mathrm{~nm})$, in the $0.18-0.19$ range used in classical buffers [38]. The light scattering detector was calibrated with toluene. The detector was controlled and the data were analyzed using the ASTRA 7.3 software (Wyatt Technology).

\section{Theoretical Background}

\subsection{SEC-MALS Experiment}

After separation of the species constituting the purified protein by HPLC on a SECcolumn and illumination by a laser with an incident intensity $I_{0}$ at a wavelength $\lambda_{0}$ of $658 \mathrm{~nm}$, the scattered light $I_{\theta}$ was recorded at three different angles. By measuring the excess Rayleigh ratio $R_{\theta}$ (the ratio of the scattered and incident light intensities at one measured angle $\theta$ ), the concentration $c$ by refractometry and adjusting the refractive index increment $d n / d c$, an absolute molar mass $M$ could be obtained using Equation (1) [2,39]. This measurement is independent of the hydrodynamic radius of the macromolecule.

$$
R_{\theta}=K^{*} M c P(\theta)\left[1-2 A_{2} M P(\theta) c\right] \text { with } K^{*}=4 \pi^{2}(d n / d c)^{2} \frac{n_{0}^{2}}{N_{a} \lambda_{0}^{4}}
$$


with $P(\theta)$, a corrective shape factor for big particles/molecules with respect to the laser wavelength [39],

$$
1 / P(\theta)=1+q^{2} \frac{R_{G}^{2}}{3}
$$

where $R_{G}$ is the gyration radius of the particle and $q$ is the scattering vector given by [39],

$$
q=4 \pi n_{0} \sin (\theta / 2) / \lambda_{0}
$$

$A_{2}$ is the second virial coefficient that is a corrective factor for a non-ideal solution and which accounts for the interaction between two particles/molecules, $n_{0}$, the refractive index of the solvent and $N_{a}$ the Avogadro number. In the case of online SEC-MALS, the $2 A_{2} M P(\theta) c$ term in Equation (1) is negligible, the orders of magnitude of $A_{2}, M$ and $c$ being $10^{-4}, 10^{5}$ and $10^{-3}$, respectively. Using Equations (2) and (3), Equation (1) becomes:

$$
K^{*} c / R_{\theta}=\frac{1}{M}\left[1+\left(16 \pi^{2} n_{0}^{2} R_{G}^{2} \sin ^{2}(\theta / 2) / 3 \lambda_{0}^{2}\right)\right]
$$

Experiments at several angles enable us to determine the molar mass, $1 / M$ being the $y$-intercept of the Zimm plot $K^{*} c / R_{\theta}=\mathrm{f}\left(\sin ^{2}(\theta / 2)\right)$. A weighted linear fit of the data requires a minimum of three detectors covering a sufficient angular range.

Concretely, in the mini-DAWN TREOS system, the intensity of scattered light $I_{\theta}$ is measured by an array of photodiodes. The laser monitor measures the laser intensity $I_{0}$ before it enters the flow cell, whereas the forward laser monitor measures the transmitted light through the flow cell and sample (Figure 1). The excess Rayleigh ratio $R_{\theta}$ of scattered to incident light intensity needs to be corrected by the detector parameters (scattering volume $V$ and distance of the scattering volume to the detector $r$ ), according to Equation (5) [40].

$$
R_{\theta}=\frac{I_{\theta} r^{2}}{I_{0} V}
$$

An accurate measurement of the laser intensity, $I_{0}$, is essential for the determination of the molar mass by SEC-MALS. In the case of macromolecules without chromophores, the variations of the intensity of the laser source due to power fluctuations are taken into account by dividing each photodiode signal by that of the laser monitor.

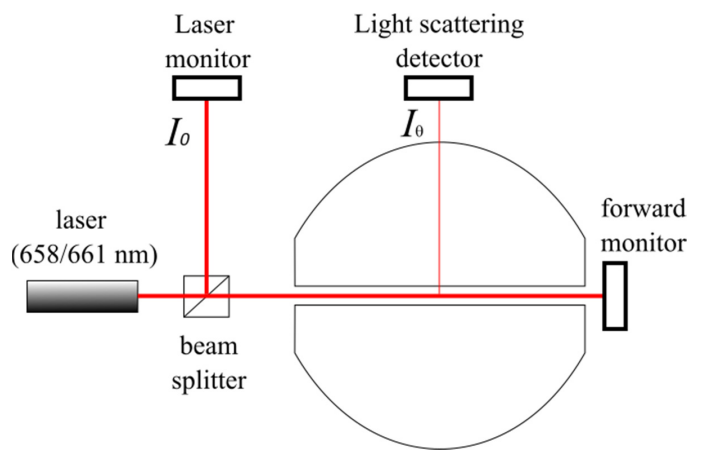

Figure 1. Schematic representation of the Mini-DAWN TREOS flow cell detectors (adapted from [40]).

\subsection{Forward Monitor Correction Mode for Samples Absorbing at the Laser Wavelength}

The Laser Monitor measures the intensity of the beam before it enters the cell, whereas the Forward Monitor measures the transmitted light through the flow cell and sample. This latter signal is used to determine the scattered intensity $I_{\text {corrected }}$ of absorbing samples. According to the Beer-Lambert law, the optical attenuation of the beam $F / F_{0}$ due to the absorbing sample, measured by the Forward Laser monitor, depends on the optical path length through the sample $L$, its concentration $c$ and its extinction coefficient $\varepsilon$. 


$$
-\log \left(F / F_{0}\right)=\varepsilon L c
$$

The scattered intensity at the center of the sample $\left(I_{\text {corrected }}\right)$ is related to the intensity of the incident laser $I_{0}$ by:

$$
-\log \left(I_{\text {corrected }} / I_{0}\right)=\varepsilon L c / 2
$$

Combining Equations (6) and (7) leads to:

$$
I_{\text {corrected }}=I_{0} \sqrt{F / F_{0}}
$$

Practically, to correct the absorption of the laser light by the samples, the ASTRA experiment file is opened with the 'MALS TREOS Configuration' and the 'forward monitor' mode is selected from the 'Divide by Laser Monitor' drop-down menu. The scattered intensity of the protein $I_{\theta}$ is obtained by subtracting the intensity scattered by the pure solvent from that of the total scattered intensity. This control is directly performed by the Astra software by subtracting the baseline ahead of the sample peak.

\section{Results}

We analyzed by SEC-MALS six [Fe-S]-binding proteins, two [2Fe-2S] regulatory proteins (CISD2 and mitoNEET) and four [4Fe-4S]-dependent enzymes (TudS, LarE, MnmA and $\mathrm{CyuA}$ ) to obtain their molar mass in solution. Both the holo- and apo-forms were investigated for each protein, except for TudS, whose apo-form could not be obtained.

\section{1. [Fe-S]-Binding Proteins Absorb at the Laser Wavelength}

The absorption spectrum of [Fe-S]-binding proteins varies with the chemical nature of the cluster [41]. The apo-proteins absorb at $280 \mathrm{~nm}$ due to their aromatic amino acids but not in the visible light spectrum. In contrast, in addition to the peak at $280 \mathrm{~nm}$, [4Fe-4S]containing proteins exhibit a maximum of absorbance around $410 \mathrm{~nm}$ (Figure 2, red and black lines). The spectrum of [2Fe-2S]-containing proteins is more complex, with several characteristic absorption maxima between 320 and $430 \mathrm{~nm}$, a peak around $470 \mathrm{~nm}$ and occasionally, a relatively broad peak between 550 and $600 \mathrm{~nm}$ (Figure 2, blue line). In both cases, the absorbance is not negligible at $658-661 \mathrm{~nm}$, which corresponds to the laser wavelength.

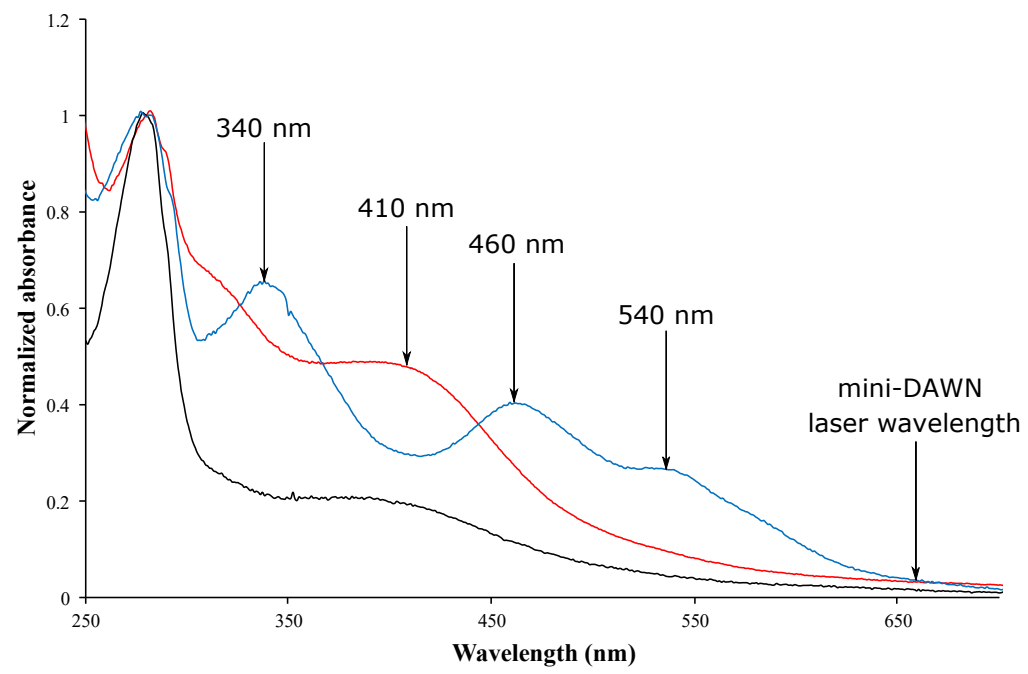

Figure 2. UV-visible absorbance spectra of the holo-forms of MnmA (black line), TudS (red line) and mitoNEET (blue line) between 250 and $700 \mathrm{~nm}$. The absorption maxima for each holo-protein and the laser wavelength are indicated by arrows. Spectra were normalized using an absorbance value of 1 at $280 \mathrm{~nm}$. 


\subsection{Corrections for Samples Absorbing at the Laser Wavelength}

Inaccurate molar masses are measured for macromolecules that absorb light at the laser wavelength $(658 / 661 \mathrm{~nm})$. In these cases, changes in measured intensity due to absorbance of the sample can be accounted for in the ASTRA software by dividing each photodiode signal by that of the forward laser monitor. Sample absorbance may be corrected by the procedure above if the forward monitor does not drop by more than approximately $20 \%$. In addition, the effect of absorbance on the absolute molar mass determination can also be prevented by increasing the laser wavelength.

For all proteins, the SEC-MALS chromatograms did not reveal peaks of high molar mass corresponding to aggregates (Figures 3 and 4). In the presence of DTT, only one main peak was observed for the apo-proteins (Figure 4A,B and data not shown). For the holo-proteins, only one main peak was also observed, as exemplified in the case of holo-TudS (Figure 3) and the NEET proteins (Figure 4C,D), indicating no aggregation.

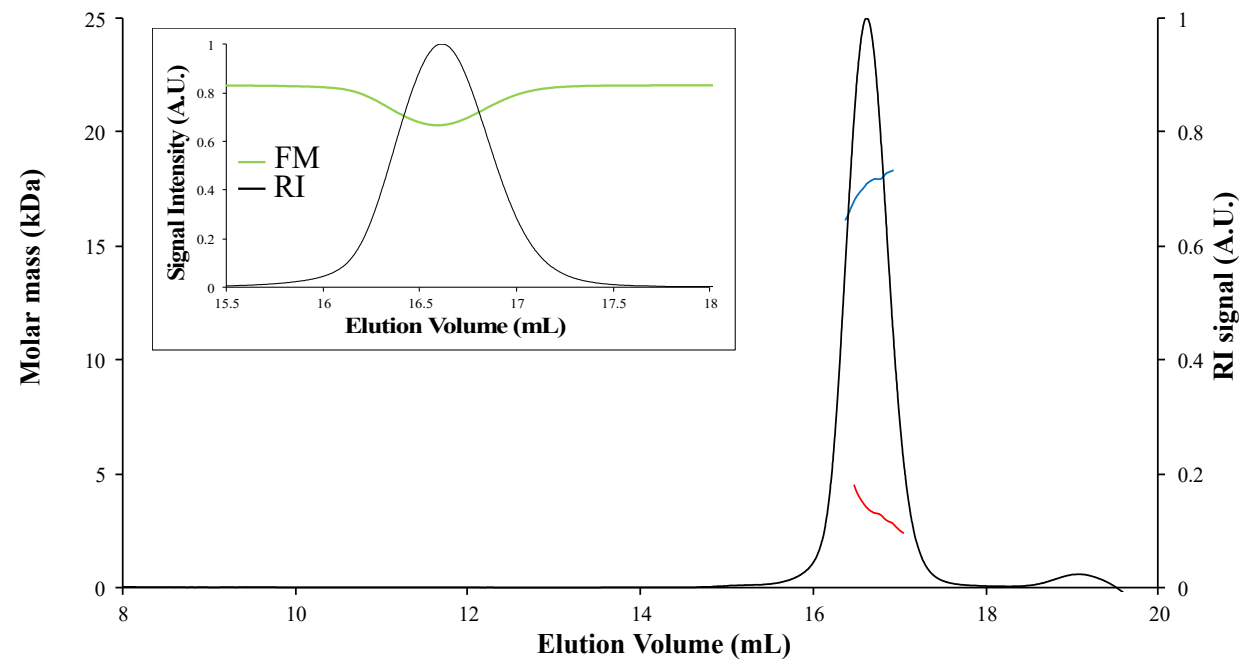

Figure 3. SEC-MALS analysis of holo-TudS. The molar mass was calculated using either the Forward Monitor (blue curve) or Laser Monitor correction modes (red curve). RI, refractive index; A.U., arbitrary unit. Insert: RI signal (black curve) and intensity signal of Forward Monitor detector (significative of protein absorbance; green curve), as a function of elution volume.

To calculate the molar mass of proteins from SEC-MALS data, one has to take into consideration the oligomerization state of the protein that is deduced by comparing the non-corrected experimental and theoretical molar mass values (Table 1). When analyzing the data of the studied apo-proteins that do not absorb light at the laser wavelength with the ASTRA software, the experimental molar masses determined without correction or using the Laser Monitor (LM) mode (normal mode for analysis of non-absorbing samples) correspond to the theoretical molar masses of the monomer or the dimer (Table 1). For the holo-proteins, the use of the Forward Monitor (FM) mode gives molar masses closer to the theoretical values than the LM mode, as shown by the significant variation in the experimentally determined molar mass, depending on whether the LM or FM modes were used. This difference was not observed with the apo-proteins, indicating that it originates from the presence of the [Fe-S] cluster and not from the protein sequence (Table 1). Therefore, the apo-proteins serve as a control (non-absorbing samples) for the validation of the method for correction of the absorbing samples. Regarding the holo-proteins, the variation of the molar mass calculated using LM or FM is exceptionally high for the TudS protein $(80 \%$ decrease in molar mass compared to the theoretical value when LM is chosen) (Figure 3), significant for CISD2 and MnmA (close to 10\%), and relatively low for LarE and CyuA (2-3\%). 
A

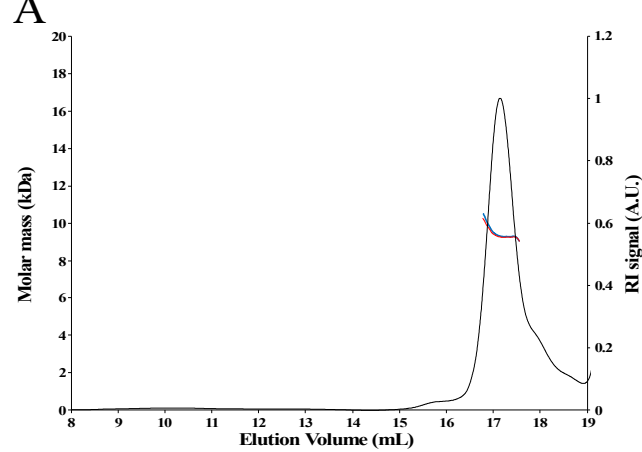

$\mathrm{C}$

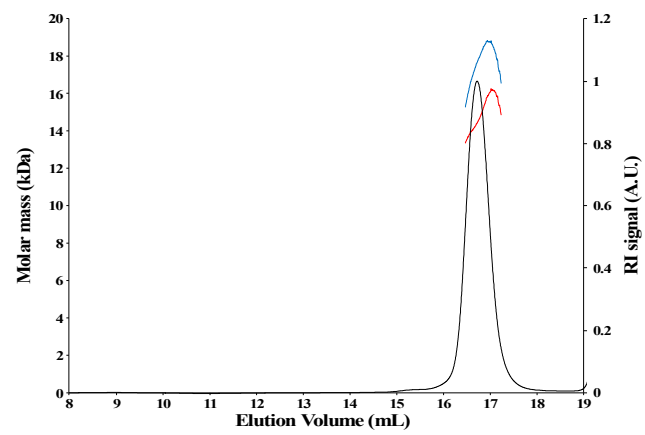

$\mathrm{B}$

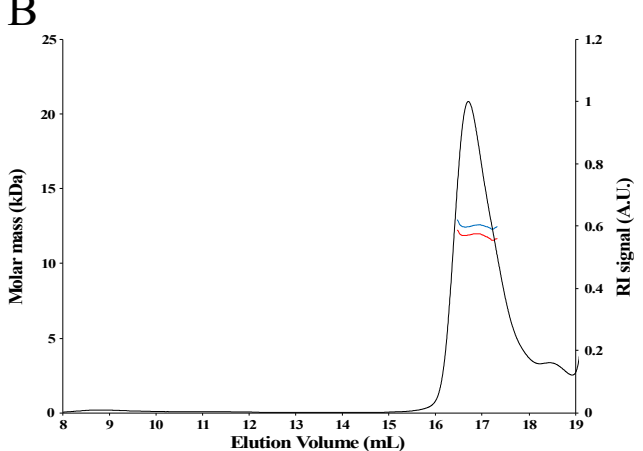

$\mathrm{D}$

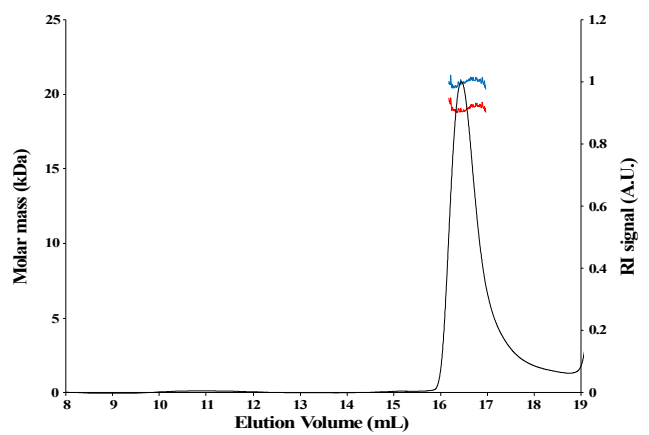

Figure 4. SEC-MALS analysis of apo- and holo-NEET proteins using either the Forward Monitor (blue curve) or Laser Monitor (red curve) modes. (A) apo-mitoNEET. (B) apo-CISD2. (C) holo-mitoNEET. (D) holo-CISD2.

Table 1. Variation of the experimentally determined molar mass using the LM and FM modes.

\begin{tabular}{|c|c|c|c|c|c|c|c|c|}
\hline \multirow[b]{2}{*}{ Protein } & \multirow[b]{2}{*}{$\begin{array}{l}\text { Theoretical } \\
\text { Molar Mass } \\
\text { (kDa) }{ }^{1}\end{array}$} & \multicolumn{7}{|c|}{ Experimental Molar Mass } \\
\hline & & & $\begin{array}{c}\text { No } \\
\text { Correction } \\
\text { (kDa) }\end{array}$ & $\begin{array}{l}\text { Oligomerization } \\
\text { State }^{1}\end{array}$ & $\begin{array}{l}\mathrm{LM}^{2} \\
(\mathrm{kDa})\end{array}$ & $\begin{array}{c}\text { Mass Difference } \\
\text { with Theoretical } \\
\text { Value Using LM }{ }^{3} \\
(\%)\end{array}$ & $\begin{array}{l}\text { FM }^{2} \\
(\mathbf{k D a})\end{array}$ & $\begin{array}{c}\text { Mass Difference } \\
\text { with Theoretical } \\
\text { Value Using FM }{ }^{3} \\
(\%)\end{array}$ \\
\hline \multirow[b]{2}{*}{ mitoNEET } & \multirow[b]{2}{*}{$8.6(\mathrm{M}) ; 17.2(\mathrm{D})$} & Apo & 9.3 & M & 9.3 & -8.1 & 9.3 & -8.1 \\
\hline & & Holo & 16.2 & $\mathrm{D}$ & 16.2 & 5.8 & 18.8 & 9.3 \\
\hline \multirow{2}{*}{ CISD2 } & \multirow{2}{*}{$11.4(\mathrm{M}) ; 22.8(\mathrm{D})$} & Apo & 11.8 & $\mathrm{M}$ & 11.9 & -4.4 & 12.6 & -10.5 \\
\hline & & Holo & 19 & $\mathrm{D}$ & 19 & 16.7 & 20.9 & 8.3 \\
\hline \multirow{2}{*}{ MnmA } & \multirow{2}{*}{$40.6(\mathrm{M})$} & Apo & 36.3 & $\mathrm{M}^{4}$ & 36.3 & 10.6 & 36.3 & 10.6 \\
\hline & & Holo & 36.1 & $\mathrm{M}$ & 36.5 & 10.1 & 40.1 & 1.2 \\
\hline \multirow{2}{*}{ LarE } & \multirow{2}{*}{$31.1(\mathrm{M}) ; 62.2(\mathrm{D})$} & Apo & 54.1 & $\mathrm{D}$ & 54.2 & 12.9 & 54.2 & 12.9 \\
\hline & & Holo & 58.8 & $\mathrm{D}$ & 58.8 & 5.5 & 60.5 & 2.7 \\
\hline \multirow{2}{*}{ CyuA } & \multirow{2}{*}{$43.7(\mathrm{M}) ; 87.4(\mathrm{D})$} & Apo & 77.9 & $\mathrm{D}$ & 77.9 & 10.9 & 78.6 & 10.1 \\
\hline & & Holo & 78.9 & $\mathrm{D}$ & 78.9 & 9.7 & 80.8 & 7.6 \\
\hline \multirow[t]{2}{*}{ TudS } & $16.5(\mathrm{M})$ & Holo & 4.5 & $\mathrm{M}$ & 3.3 & 80.0 & 17.9 & -8.5 \\
\hline & & $\begin{array}{l}\text { M, mor } \\
\text { lonitor } \\
\text { onome } \\
\text { e prese }\end{array}$ & $\begin{array}{l}\text { omer; } \mathrm{D}, \mathrm{dim} \\
\text { mode (that } \mathrm{c} \\
\mathrm{r} \text { theoretical } \\
\text { nce of } 5 \mathrm{mM}\end{array}$ & $\begin{array}{l}\text { Molar mass deter } \\
\text { cts for absorbing s } \\
\text { e } \times N \text {, with } N=1 \\
\Gamma \text {. In the absence of }\end{array}$ & $\begin{array}{l}\text { ned usir } \\
\text { aples). } \\
\text { tonome } \\
\text { TT, the }\end{array}$ & $\begin{array}{l}\text { the Laser Monitor } \mathrm{m} \\
\text { monomer theoretical } \\
\text { or } \mathrm{N}=2 \text { (dimer). }{ }^{4} \\
\text { dimer increased to } 4\end{array}$ & $\begin{array}{l}\text { e (norm } \\
\text { alue } \times 1 \\
\% \text { dimer } \\
\% \text { [32]. }\end{array}$ & $\begin{array}{l}\text { mode) or the Forwa } \\
\text { - LM (or FM) value } \\
\text { form was observed }\end{array}$ \\
\hline
\end{tabular}

These variations seem to correlate with the differences between the theoretical value of the mass extinction coefficient at $280 \mathrm{~nm}$ (obtained using the ProtParam webserver https: / / web.expasy.org/protparam/ accessed on 2 February 2022) and the experimental value of the mass extinction coefficient obtained using the ASTRA " $U V$-extinction from RI peak" method (Table 2). For the apo-proteins, the experimental and theoretical extinction coefficients values are very close to each other, which is not the case for the holo-proteins. 
In particular, the huge difference between the theoretical and experimental extinction coefficients for holo-TudS remains unexplained.

Table 2. Comparison of the theoretical and experimental extinction coefficients at $280 \mathrm{~nm}$ for the six studied [Fe-S] proteins.

\begin{tabular}{|c|c|c|c|}
\hline \multicolumn{2}{|c|}{ Protein Name } & \multirow{3}{*}{$\begin{array}{c}\text { Theoretical Extinction } \\
\text { Coefficient } \mathrm{mL} / \mathrm{mg} \cdot \mathrm{cm}\end{array}$} & \multirow{3}{*}{$\begin{array}{c}\begin{array}{c}\text { Experimental Extinction } \\
\text { Coefficient } \mathbf{~} \mathrm{L} / \mathrm{mg} \cdot \mathrm{cm}\end{array} \\
1.545 \\
0.846\end{array}$} \\
\hline & Holo & & \\
\hline mitoNEET & Apo & & \\
\hline \multirow{2}{*}{ CISD2 } & Holo & \multirow[b]{2}{*}{0.616} & 1.2262 \\
\hline & Apo & & 0.768 \\
\hline \multirow{2}{*}{$\mathrm{MnmA}$} & Holo & \multirow{2}{*}{0.993} & 1.46 \\
\hline & Apo & & 1.1 \\
\hline \multirow{2}{*}{ LarE } & Holo & \multirow{2}{*}{$0.473^{1}$} & $0.638^{2}$ \\
\hline & Apo & & 0.565 \\
\hline \multirow{2}{*}{ CyuA } & Holo & \multirow{2}{*}{0.583} & 0.858 \\
\hline & Apo & & 0.733 \\
\hline TudS & Holo & 0.514 & 1.406 \\
\hline
\end{tabular}

\section{Discussion}

Our SEC-MALS analysis indicates that both human NEET proteins, mitoNEET and CISD2, are monomeric in their apo-forms but dimeric in their holo-forms. We thus confirm here that the [2Fe-2S] cluster is essential for the stabilization of the dimer of both NEET proteins. We had previously shown that the cluster is essential for the folding of the mitoNEET [42] and CISD2 [26] protomers. Indeed, the loss of the cluster leads to incorrect folding of the protomer and, consequently, to the loss of protein dimerization. Moreover, native mass spectrometry showed that holo-mitoNEET is a dimer but not apo-mitoNEET [29]. It is interesting to note that although the [2Fe-2S] cluster of the two NEET proteins is essential for dimerization, it is not located at the interface between the monomers, as shown by the crystal structures of mitoNEET [43-45] and CISD2 [46]. In contrast to the NEET proteins, LarE and CyuA are intrinsically dimeric because both the apo-and holo-forms are dimers, indicating that the cluster is not crucial for the correct folding of these proteins.

This study reveals the power of SEC-MALS to investigate the involvement of an [Fe-S] cluster in maintaining the oligomerization state of a protein. The binding of a [2Fe-2S] cluster at the dimer interface has been reported for several proteins. Human mitochondrial ISCU2 is the scaffold protein of the ISC (iron-sulfur cluster assembly) complex involved in the synthesis of [Fe-S] clusters in living cells and the subsequent transfer of clusters to target apoproteins. ISCU2 synthesizes a bridging [2Fe-2S] cluster by assembling two ISCU2 monomers. It has recently been explained how, after iron and sulfur loading onto monomeric ISCU2, the interaction between tyrosines, belonging to two different monomers, triggers ISCU2 dimerization and the generation of a bridging [2Fe-2S] cluster [47]. Similarly, human cytosolic monothiol glutaredoxin-3 (GLRX3) is a protein essential for the maturation of cytosolic [4Fe-4S] proteins. Dimeric cluster-bridged GLRX3 transfers its [2Fe-2S] clusters to human NUBP1, an essential early component of the cytosolic iron-sulfur assembly (CIA) machinery [48]. Determining the molar mass of both the apo- and holo-forms of such proteins is essential to shed light on the structural function of the cluster.

\section{Conclusions}

SEC-MALS is a valuable technique to determine the molar mass in solution of proteins in their native state, which does not require desolvation of the sample or very low salt concentrations. The Wyatt miniDAWN TREOS instrument can be used to determine the 
absolute molar mass of proteins, even when they exhibit absorption at the laser wavelength, as is the case for the [Fe-S]-containing proteins discussed in this article, by using the Forward Monitor instead of the Laser Monitor mode. At present, the MALS devices on the market, Viscotek TDA 305 and OMNISEC REVEAL from Malvern Panalytical (Malvern, United Kingdom) or LenS3 from Tosoh Bioscience (King of Prussia, PA, USA), do not provide the possibility to correct for absorption of samples at the laser wavelength. Obtaining the holo-form of these proteins is often difficult because the cluster can be easily destroyed by oxygen, so working under anaerobic conditions is required. In our study, the holo-proteins with labile clusters were purified and frozen anaerobically, and SEC-MALS analysis was performed aerobically immediately after thawing. Whereas it is tempting to determine only the molar mass of the apo-protein, we show here that also determining the molar mass of the holo-protein is crucial for understanding potential changes in oligomerization states upon cluster binding, which may be crucial for the function of the protein, as shown in the case of the [2Fe-2S]-containing NEET proteins.

Author Contributions: Conceptualization, C.V. and B.G.-P.; methodology, C.V.; software, C.V.; validation, C.V., M.-P.G.-C. and B.G.-P.; formal analysis, C.V.; investigation, C.V., J.Z., P.Z., N.H. and M.S.; writing-original draft preparation, C.V. and B.G.-P.; writing-review and editing, C.V., M.-P.G.-C. and B.G.-P.; visualization, C.V.; supervision, B.G.-P. All authors have read and agreed to the published version of the manuscript.

Funding: This work was supported by the French State Program 'Investissements d'Avenir' (Grants "LABEX DYNAMO", ANR-11-LABX-0011).

Institutional Review Board Statement: Not applicable.

Informed Consent Statement: Not applicable.

Data Availability Statement: Not applicable.

Acknowledgments: We warmly thank Thierry Azoulay from Wyatt technology for sharing his expertise and giving us fruitful advice. We also thank Charlotte Cabanne, Gilles Joucla and Majid Noubhani for technical support with the ENSTBB SEC-MALS apparatus. This work has also benefited from the facilities and expertise of the Macromolecular Interaction Platforms of I2BC.

Conflicts of Interest: The authors declare no conflict of interest.

\section{References}

1. Uliyanchenko, E. Size-exclusion chromatography-from high-performance to ultra-performance. Anal. Bioanal. Chem. 2014, 406, 6087-6094. [CrossRef]

2. Wyatt, P.J. Light scattering and the absolute characterization of macromolecules. Anal. Chim. Acta 1993, 272, 1-40. [CrossRef]

3. Wen, J.; Arakawa, T.; Philo, J.S. Size-exclusion chromatography with on-line light-scattering, absorbance, and refractive index detectors for studying proteins and their interactions. Anal. Biochem. 1996, 240, 155-166. [CrossRef]

4. Adawy, A.; Groves, M.R. The Use of Size Exclusion Chromatography to Monitor Protein Self-Assembly. Crystals 2017, 7, 331. [CrossRef]

5. Some, D.; Amartely, H.; Tsadok, A.; Lebendiker, M. Characterization of Proteins by Size-Exclusion Chromatography Coupled to Multi-Angle Light Scattering (SEC-MALS). J. Vis. Exp. 2019, 148, e59615. [CrossRef]

6. Hardouin, P.; Velours, C.; Bou-Nader, C.; Assrir, N.; Laalami, S.; Putzer, H.; Durand, D.; Golinelli-Pimpaneau, B. Dissociation of the Dimer of the Intrinsically Disordered Domain of RNase Y upon Antibody Binding. Biophys. J. 2018, 115, 2102-2113. [CrossRef] [PubMed]

7. Serebryany, E.; Folta-Stogniew, E.; Liu, J.; Yan, E.C. Homodimerization enhances both sensitivity and dynamic range of the ligand-binding domain of type 1 metabotropic glutamate receptor. FEBS Lett. 2016, 590, 4308-4317. [CrossRef]

8. Proença, D.; Velours, C.; Leandro, C.; Garcia, M.; Pimentel, M.; Sao-Jose, C. A two-component, multimeric endolysin encoded by a single gene. Mol. Microbiol. 2015, 95, 739-753. [CrossRef] [PubMed]

9. Loiseau, L.; Fyfe, C.; Aussel, L.; Hajj Chehade, M.; Hernandez, S.B.; Faivre, B.; Hamdane, D.; Mellot-Draznieks, C.; Rascalou, B.; Pelosi, L.; et al. The UbiK protein is an accessory factor necessary for bacterial ubiquinone (UQ) biosynthesis and forms a complex with the UQ biogenesis factor UbiJ. J. Biol. Chem. 2017, 292, 11937-11950. [CrossRef] [PubMed]

10. Hastie, K.; Rayaprolu, V.; Saphire, E.O. Analysis of Oligomeric and Glycosylated Proteins by Size-Exclusion Chromatography Coupled with Multiangle Light Scattering. Methods Mol. Biol. 2021, 2271, 343-359. [CrossRef] 
11. Zinovyev, G.; Sulaeva, I.; Podzimek, S.; Rössner, D.; Kilpeläinen, I.; Sumerskii, I.; Rosenau, T.; Potthast, A. Getting Closer to Absolute Molar Masses of Technical Lignins. ChemSusChem 2018, 11, 3259-3268. [CrossRef] [PubMed]

12. Andreini, C.; Rosato, A.; Banci, L. The relationship between environmental dioxygen and iron-sulfur proteins explored at the genome level. PLoS ONE 2017, 12, e0171279. [CrossRef] [PubMed]

13. Beinert, H. Iron-sulfur proteins: Ancient structures, still full of surprises. J. Biol. Inorg. Chem. 2000, 5, 2-15. [CrossRef]

14. Dinis, P.; Wieckowski, B.M.; Roach, P.L. Metallocofactor assembly for [FeFe]-hydrogenases. Curr. Opin. Struct. Biol. 2016, 41, 90-97. [CrossRef]

15. Jeoung, J.H.; Martins, B.M.; Dobbek, H. Double-Cubane [8Fe9S] Clusters: A Novel Nitrogenase-Related Cofactor in Biology. Chembiochem 2020, 21, 1710-1716. [CrossRef]

16. Yakovlev, G.; Reda, T.; Hirst, J. Reevaluating the relationship between EPR spectra and enzyme structure for the iron sulfur clusters in NADH:quinone oxidoreductase. Proc. Natl. Acad. Sci. USA 2007, 104, 12720-12725. [CrossRef] [PubMed]

17. Crack, J.C.; Green, J.; Thomson, A.J.; Le Brun, N.E. Iron-sulfur clusters as biological sensors: The chemistry of reactions with molecular oxygen and nitric oxide. Acc. Chem. Res. 2014, 47, 3196-3205. [CrossRef]

18. Bouton, C.; Drapier, J.C. Iron regulatory proteins as NO signal transducers. Sci. STKE 2003, 2003, pe17. [CrossRef] [PubMed]

19. Crack, J.C.; Green, J.; Thomson, A.J.; Le Brun, N.E. Iron-sulfur cluster sensor-regulators. Curr. Opin. Chem. Biol. 2012, 16, 35-44. [CrossRef] [PubMed]

20. Mettert, E.L.; Kiley, P.J. Fe-S proteins that regulate gene expression. Biochim. Biophys. Acta 2015, 1853, 1284-1293. [CrossRef]

21. Crack, J.C.; Jervis, A.J.; Gaskell, A.A.; White, G.F.; Green, J.; Thomson, A.J.; Le Brun, N.E. Signal perception by FNR: The role of the iron-sulfur cluster. Biochem. Soc. Trans. 2008, 36, 1144-1148. [CrossRef]

22. Mettert, E.L.; Kiley, P.J. Reassessing the Structure and Function Relationship of the $\mathrm{O}_{2}$ Sensing Transcription Factor FNR. Antioxid. Redox Signal. 2018, 29, 1830-1840. [CrossRef]

23. Flint, D.H.; Allen, R.M. Iron-Sulfur Proteins with Nonredox Functions. Chem. Rev. 1996, 96, 2315-2334. [CrossRef]

24. Beinert, H.; Kennedy, M.C.; Stout, C.D. Aconitase as Iron-Sulfur Protein, Enzyme, and Iron-Regulatory Protein. Chem. Rev. 1996, 96, 2335-2374. [CrossRef] [PubMed]

25. Castro, L.; Tórtora, V.; Mansilla, S.; Radi, R. Aconitases: Non-redox Iron-Sulfur Proteins Sensitive to Reactive Species. Acc. Chem. Res. 2019, 52, 2609-2619. [CrossRef]

26. Salameh, M.; Riquier, S.; Guittet, O.; Huang, M.E.; Vernis, L.; Lepoivre, M.; Golinelli-Cohen, M.P. New Insights of the NEET Protein CISD2 Reveals Distinct Features Compared to Its Close Mitochondrial Homolog mitoNEET. Biomedicines 2021, 9, 384. [CrossRef]

27. Karmi, O.; Marjault, H.B.; Pesce, L.; Carloni, P.; Onuchic, J.N.; Jennings, P.A.; Mittler, R.; Nechushtai, R. The unique fold and lability of the [2Fe-2S] clusters of NEET proteins mediate their key functions in health and disease. J. Biol. Inorg. Chem. 2018, 23, 599-612. [CrossRef] [PubMed]

28. Golinelli-Cohen, M.-P.; Lescop, E.; Mons, C.; Goncalves, S.; Clemancey, M.; Santolini, J.; Guittet, E.; Blondin, G.; Latour, J.M.; Bouton, C. Redox Control of the Human Iron-Sulfur Repair Protein MitoNEET Activity via Its Iron-Sulfur Cluster. J. Biol. Chem. 2016, 291, 7583-7593. [CrossRef]

29. Mons, C.; Botzanowski, T.; Nikolaev, A.; Hellwig, P.; Cianférani, S.; Lescop, E.; Bouton, C.; Golinelli-Cohen, M.-P. The $\mathrm{H}_{2} \mathrm{O}_{2}-$ resistant Fe-S redox switch mitoNEET acts as a pH sensor to repair stress-damaged Fe-S protein. Biochemistry 2018, 57, 5616-5628. [CrossRef]

30. Chen, M.; Asai, S.I.; Narai, S.; Nambu, S.; Omura, N.; Sakaguchi, Y.; Suzuki, T.; Ikeda-Saito, M.; Watanabe, K.; Yao, M.; et al. Biochemical and structural characterization of oxygen-sensitive 2-thiouridine synthesis catalyzed by an iron-sulfur protein TtuA. Proc. Natl. Acad. Sci. USA 2017, 114, 4954-4959. [CrossRef] [PubMed]

31. Arragain, S.; Bimai, O.; Legrand, P.; Caillat, S.; Ravanat, J.L.; Touati, N.; Binet, L.; Atta, M.; Fontecave, M.; Golinelli-Pimpaneau, B. Nonredox thiolation in tRNA occurring via sulfur activation by a [4Fe-4S] cluster. Proc. Natl. Acad. Sci. USA 2017, 114, 7355-7360. [CrossRef]

32. Zhou, J.; Lénon, M.; Touati, N.; Ravanat, J.-L.; Velours, C.; Fontecave, M.; Barras, F.; Golinelli-Pimpaneau, B. Iron sulfur biology invades tRNA modification: The case of U34 sulfuration. Nucleic Acids Res. 2021, 49, 3997-4007. [CrossRef]

33. Shigi, N. Biosynthesis and Degradation of Sulfur Modifications in tRNAs. Int. J. Mol. Sci. 2021, 22, 11937. [CrossRef]

34. Fellner, M.; Desguin, B.; Hausinger, R.P.; Hu, J. Structural insights into the catalytic mechanism of a sacrificial sulfur insertase of the N-type ATP pyrophosphatase family, LarE. Proc. Natl. Acad. Sci USA 2017, 114, 9074-9079. [CrossRef]

35. Zhou, J.; Pecqueur, L.; Aučynaitè, A.; Fuchs, J.; Rutkienė, R.; Vaitekūnas, J.; Meškys, R.; Boll, M.; Fontecave, M.; Urbonavičius, J.; et al. Structural evidence for a [4Fe-5S] intermediate in the non-redox desulfuration of thiouracil. Angew. Chem. Int. Ed. Engl. 2021, 60, 424-431. [CrossRef]

36. Tchong, S.I.; Xu, H.; White, R.H. L-cysteine desulfidase: An [4Fe-4S] enzyme isolated from Methanocaldococcus jannaschii that catalyzes the breakdown of L-cysteine into pyruvate, ammonia, and sulfide. Biochemistry 2005, 44, 1659-1670. [CrossRef] [PubMed]

37. Mons, C.; Ferecatu, I.; Riquier, S.; Lescop, E.; Bouton, C.; Golinelli-Cohen, M.-P. Combined biochemical, biophysical and cellular methods to study Fe-S cluster transfer and cytosolic aconitase repair by mitoNEET. Methods Enzymol. 2017, 595, 83-108. [CrossRef] [PubMed] 
38. Zhao, H.; Brown, P.H.; Schuck, P. On the distribution of protein refractive index increments. Biophys. J. 2011, 100, $2309-2317$. [CrossRef] [PubMed]

39. Lorber, B. Analytical light scattering methods in molecular and structural biology: Experimental aspects and results. arXiv 2018, arXiv:1810.00611.

40. Technical Note TN3000, Wyatt Technology, Calibration and Normalization of MALS Detectors. Available online: https: / / chem.uiowa.edu/sites / chem.uiowa.edu/files / groups /TN3000\%20-\%20Calibration \%20and\%20Normalization \%20of $\% 20$ MALS\%20Detectors\%20Rev\%20A.pdf (accessed on 2 February 2022).

41. Freibert, S.A.; Weiler, B.D.; Bill, E.; Pierik, A.J.; Mühlenhoff, U.; Lill, R. Biochemical Reconstitution and Spectroscopic Analysis of Iron-Sulfur Proteins. Methods Enzymol. 2018, 599, 197-226. [CrossRef]

42. Ferecatu, I.; Goncalves, S.; Golinelli-Cohen, M.P.; Clemancey, M.; Martelli, A.; Riquier, S.; Guittet, E.; Latour, J.M.; Puccio, H.; Drapier, J.C.; et al. The diabetes drug target MitoNEET governs a novel trafficking pathway to rebuild an Fe-S cluster into cytosolic aconitase/iron regulatory protein 1. J. Biol. Chem. 2014, 289, 28070-28086. [CrossRef]

43. Paddock, M.L.; Wiley, S.E.; Axelrod, H.L.; Cohen, A.E.; Roy, M.; Abresch, E.C.; Capraro, D.; Murphy, A.N.; Nechushtai, R.; Dixon, J.E.; et al. MitoNEET is a uniquely folded $2 \mathrm{Fe} 2 \mathrm{~S}$ outer mitochondrial membraneprotein stabilized by pioglitazone. Proc. Natl. Acad. Sci. USA 2007, 104, 14342-14347. [CrossRef] [PubMed]

44. Hou, X.; Liu, R.; Ross, S.; Smart, E.J.; Zhu, H.; Gong, W. Crystallographic studies of human MitoNEET. J. Biol. Chem. 2007, 282, 33242-33246. [CrossRef]

45. Lin, J.; Zhou, T.; Ye, K.; Wang, J. Crystal structure of human mitoNEET reveals distinct groups of iron sulfur proteins. Proc. Natl. Acad. Sci. USA 2007, 104, 14640-14645. [CrossRef] [PubMed]

46. Tamir, S.; Eisenberg-Domovich, Y.; Conlan, A.R.; Stofleth, J.T.; Lipper, C.H.; Paddock, M.L.; Mittler, R.; Jennings, P.A.; Livnah, O.; Nechushtai, R.A. A point mutation in the [2Fe-2S] cluster binding region of the NAF-1 protein (H114C) dramatically hinders the cluster donor properties. Acta Crystallogr. D Biol. Crystallogr. 2014, 70, 1572-1578. [CrossRef] [PubMed]

47. Freibert, S.A.; Boniecki, M.T.; Stümpfig, C.; Schulz, V.; Krapoth, N.; Winge, D.R.; Mühlenhoff, U.; Stehling, O.; Cygler, M.; Lill, R. $\mathrm{N}$-terminal tyrosine of ISCU2 triggers [2Fe-2S] cluster synthesis by ISCU2 dimerization. Nat. Commun. 2021, 12, 6902. [CrossRef] [PubMed]

48. Camponeschi, F.; Prusty, N.R.; Heider, S.A.E.; Ciofi-Baffoni, S.; Banci, L. GLRX3 Acts as a [2Fe-2S] Cluster Chaperone in the Cytosolic Iron-Sulfur Assembly Machinery Transferring [2Fe-2S] Clusters to NUBP1. J. Am. Chem. Soc. 2020, 142. [CrossRef] [PubMed] 